\title{
On Bounded Truth-Table, Conjunctive, and Randomized Reductions to Sparse Sets
}

\author{
Vikraman Arvind* \\ Department of Computer Science and Engineering \\ Indian Institute of Technology, Delhi \\ New Delhi 110016, India \\ Johannes Köbler and Martin Mundhenk \\ Abteilung für Theoretische Informatik \\ Universität Ulm \\ Oberer Eselsberg \\ D-W-7900 Ulm, Germany
}

February 11, 1993

\begin{abstract}
In this paper we study the consequences of the existence of sparse hard sets for $\mathrm{NP}$ and other complexity classes under certain types of deterministic, randomized, and nondeterministic reductions. We show that if an NP-complete set is bounded truth-table reducible to some set that conjunctively reduces to a sparse set then $\mathrm{P}=$ NP. This result subsumes and extends previously known results [?, ?, ?] yielding a collapse of PH to P under the assumption that NP has sparse hard sets. Relatedly, we show that if an NP-complete set is bounded truth-table reducible to some set that randomly reduces (via a co-rp reduction) to some set that conjunctively reduces to a sparse set then RP $=$ NP. We also prove similar results under the (apparently) weaker assumption that some solution of the promise problem (1SAT, SAT) reduces via the mentioned reductions to a sparse set. Our proofs are obtained by combining the left set technique [?] with the Hausdorff representation for sets in the boolean closure of set rings.

Finally we consider nondeterministic polynomial time many-one reductions to sparse and co-sparse sets. We prove that if a coNP-complete set reduces via a nondeterministic polynomial time many-one reduction to a co-sparse set then $\mathrm{PH}=$ $\Theta_{2}^{p}$. On the other hand, we show that nondeterministic polynomial time many-one reductions to sparse sets are as powerful as nondeterministic Turing reductions to sparse sets.
\end{abstract}

\footnotetext{
${ }^{*}$ Work done while visiting Universität Ulm. Supported in part by an Alexander von Humboldt research fellowship.
} 


\section{Introduction}

Sparse sets play a central role in structural complexity theory. An important line of research that has been very fruitful is the study of sparse hard sets under different kinds of reductions. This line of research opened with the question whether there can possibly exist sparse complete sets for NP under polynomial time many-one reductions (it was conjectured by L. Berman and J. Hartmanis [?] that there are no sparse NP-complete sets).

The first results were P. Berman's proof that $\mathrm{P}=\mathrm{NP}$ if some tally set is NP-complete [?] and Fortune's proof that if there is a sparse coNP-complete set, then $\mathrm{P}=\mathrm{NP}[?]$. Mahaney settled the 'sparseness' conjecture by proving that if any NP-complete set manyone reduces to a sparse set then $\mathrm{P}=\mathrm{NP}[?]$. From an entirely different angle of research, the possible existence of sparse Turing-hard sets for NP was studied in [?]. This question is equivalent to NP-complete problems having nonuniform polynomial-size circuits. Karp, Lipton, and Sipser proved that if NP has sparse Turing-hard sets then the polynomial hierarchy collapses to $\Sigma_{2}^{p}[?]$.

Discovering consequences of the existence of sparse complete sets for different kinds of truth-table reducibilities has remained an active research area. The next important advance was made recently by Ogiwara and Watanabe [?] when they proved using a new left-set technique that if NP has sparse hard sets under polynomial time bounded truthtable reductions then $\mathrm{P}=\mathrm{NP}$. More recently, this has been followed up by similar results for polynomial time conjunctive truth-table reductions [?, ?], and in [?] even for more flexible truth-table reductions (e.g. bounded conjunctive reductions to the 1-truth-table closure of the conjunctive closure of sparse sets). These results demonstrate the efficacy of the left-set technique introduced in [?] (in fact, the older result of Mahaney has now a considerably easier proof).

The main results of this paper concern the existence of sparse hard sets for NP and other complexity classes under certain types of deterministic, randomized, and nondeterministic reductions. In Section ?? we prove that if NP $\subseteq R_{b t t}^{p}\left(R_{c t t}^{p}(\mathrm{SPARSE})\right)$ then $\mathrm{P}=$ NP. This result subsumes and extends all previously known results on reductions of NP sets to sparse sets via various types of polynomial time truth-table reductions that yield a collapse of the polynomial hierarchy to $\mathrm{P}$.

In Section ?? we consider randomized reductions to sparse sets and show that if $\mathrm{NP} \subseteq R_{b t t}^{p}\left(R_{m}^{c 0^{-r p}}\left(R_{c t t}^{p}(\mathrm{SPARSE})\right)\right)$ then RP $=$ NP. (D. Ranjan and P. Rohatgi [?] have independently shown that if $\mathrm{NP} \subseteq R_{m}^{c o-r p}(\mathrm{SPARSE})$ then $\mathrm{RP}=\mathrm{NP}$.)

Relatedly, we show in Section ?? that if the promise problem (1SAT, SAT) has a solution in $R_{b t t}^{p}\left(R_{c t t}^{p}(\mathrm{SPARSE})\right)$ then it has a solution in $\mathrm{P}$. We also show that the conclusion $\mathrm{RP}=\mathrm{NP}$ can be derived from the assumption that the promise problem (1SAT, SAT) has a solution in $R_{b t t}^{p}\left(R_{m}^{c 0^{-r p}}\left(R_{c t t}^{p}(\mathrm{SPARSE})\right)\right)$.

The technique used in our proofs is novel. It combines the method of left sets with a classical representation theorem due to Hausdorff for sets in the boolean closure of set rings, i.e. classes of sets closed under union and intersection. Since the reduction classes to sparse sets that we consider are set rings and since the bounded truth-table closure of these classes coincides with the boolean closure, sets in the bounded truth-table closure of these classes have a Hausdorff representation. The internal structure of left sets combined with the structure imposed by a Hausdorff representation for it plays a crucial role in designing efficient decision procedures for such sets. The Hausdorff representation was first used 
in a complexity theoretic context by Wechsung and Wagner [?] where they relate the bounded truth-table closure of NP to the boolean hierarchy over NP.

In Section ?? we do a trade-off analysis for our algorithms regarding the density of the set reduced to and the power of the reduction used.

Pursuing the question of existence of sparse hard sets further (in Section ??), we consider nondeterministic reductions (as defined by Ladner, Lynch, and Selman [?]) and show that if a coNP-complete set can be reduced via a nondeterministic reduction to a cosparse set then the polynomial hierarchy collapses to $\Theta_{2}^{p}$. A similar result seems unlikely for sparse sets since (as we show) nondeterministic many-one reductions to sparse sets surprisingly turn out to be as powerful as nondeterministic Turing reductions to sparse sets. We also prove that if $\Sigma_{2}^{p}$ is bounded truth-table reducible to a set that can be reduced via a nondeterministic reduction to a co-sparse set then the polynomial hierarchy collapses to $\Delta_{2}^{p}$.

\section{Preliminaries and notation}

A set $T$ is called a tally set if $T \subseteq 0^{*}$. The census function of a set $A$ is census ${ }_{A}(n)=\left|A^{\leq n}\right|$. A set $S$ is called sparse if its census function is bounded above by a polynomial. We use TALLY and SPARSE to represent, respectively, the classes of tally and sparse sets. For a class $\mathcal{K}$ of sets we denote the union of all sets in $\mathcal{K}$ by $\cup \mathcal{K}$. Let $\langle\cdot, \cdot\rangle$ denote a standard pairing function.

The reductions discussed in this paper are the polynomial-bounded reductions defined by Ladner, Lynch, and Selman [?] and by Adleman and Manders [?].

Notation [?] For any reducibility $\leq_{r}^{\alpha}$ and any class of sets $\mathcal{C}$, let $R_{r}^{\alpha}(\mathcal{C})=\left\{A \mid A \leq_{r}^{\alpha} B\right.$ for some set $B \in \mathcal{C}\}$, where $\alpha \in\{p, n p, c o-n p, r p, c o-r p\}$ and $r \in\{m, c, d, b, T\}$.

Definition 2.1 The join of two sets $A$ and $B$, denoted $A \oplus B$, is defined as

$$
A \oplus B=\{0 x \mid x \in A\} \cup\{1 x \mid x \in B\}
$$

Definition 2.2 A class $\mathcal{K}$ of sets that includes $\emptyset$ and $\Sigma^{*}$ and is closed under finite unions and finite intersections is said to be a set ring.

Definition 2.3 [?] For a set $A$ in NP let $P_{A} \in \mathrm{P}$ and $q$ be a polynomial such that $A=\left\{x \mid\left(\exists w \in \Sigma^{q(|x|)}\right)\left[\langle x, w\rangle \in P_{A}\right]\right\}$. For $x \in A$ let $w_{\max }(x)=\max \left\{w \in \Sigma^{q(|x|)} \mid\right.$ $\left.\langle x, w\rangle \in P_{A}\right\}$. Then Left $(A)=\left\{\langle x, w\rangle \mid x \in A, w \in \Sigma^{q(|x|)}\right.$ and $\left.w \leq w_{\max }(x)\right\}$ is the left set of $A$.

Note that the left set depends on the particular witness relation $P_{A}$.

\section{Bounded truth-tables on conjunctive reductions to sparse sets}

The main result of this section is that if $\mathrm{NP} \subseteq R_{b}^{p}\left(R_{c t t}^{p}(\mathrm{SPARSE})\right)$ then $\mathrm{P}=\mathrm{NP}$. This result subsumes and extends all previously known results on reductions of NP sets to sparse sets via various types of polynomial time truth-table reductions that yield a collapse 
of $\mathrm{PH}$ to $\mathrm{P}$. We also discuss similar consequences for the classes PP, $\mathrm{C}_{=} \mathrm{P}$, FewP, Few, and UP.

The following characterization of the boolean closure of set rings due to Hausdorff plays a key role in many results of this paper.

Theorem 3.1 [?, ?] Let $\mathcal{K}$ be a set ring and let $B C(\mathcal{K})$ be the closure of $\mathcal{K}$ under finite union, finite intersection, and complement. Then every $A \in B C(\mathcal{K})$ can be represented as $A=\bigcup_{i=1}^{k}\left(A_{2 i-1}-A_{2 i}\right)$, where $A_{j} \in K, 1 \leq j \leq 2 k$, and $A_{1} \supseteq A_{2} \supseteq \cdots \supseteq A_{2 k}$.

In order to obtain a Hausdorff representation for sets in $R_{b}^{p}\left(R_{c t t}^{p}\right.$ (SPARSE)) we need to show that $R_{c t t}^{p}($ SPARSE) is a set ring.

Lemma $3.2 R_{c t t}^{p}($ SPARSE) is a set ring.

Proof $R_{c t t}^{p}($ TALLY) is easily seen to be closed under finite unions and intersections. A recent result of Buhrman, Longpré, and Spaan [?] showing that SPARSE $\subset R_{c t t}^{p}($ TALLY) implies $R_{c t t}^{p}(\mathrm{SPARSE})=R_{c t t}^{p}(\mathrm{TALLY})$. Hence, $R_{c t t}^{p}(\mathrm{SPARSE})$ is a set ring.

Fact 3.3 If a class $\mathcal{K}$ of sets contains a set different from $\emptyset$ and $\Sigma^{*}$ and is closed under join and polynomial time many-one reductions then $R_{b}^{p}(\mathcal{K})=B C(\mathcal{K})$.

Remark All the reduction classes to sparse sets considered in this paper fulfil the conditions to apply Fact??.

Theorem 3.4 If $A \in \mathrm{NP}$ such that Left $(A) \in R_{b}^{p}\left(R_{c t t}^{p}(\mathrm{SPARSE})\right)$ then $A \in \mathrm{P}$.

Proof Let $q$ be a polynomial and let $P_{A}$ be a polynomial-time computable set such that $A=\left\{x \mid\left(\exists w \in \Sigma^{q(|x|)}\right)\left[\langle x, w\rangle \in P_{A}\right]\right\}$. Recall that Left $(A)=\{\langle x, w\rangle \mid x \in A \wedge w \in$ $\left.\Sigma^{q(|x|)} \wedge w \leq w_{\max }\right\}$, where $w_{\max }=\max \left\{w \in \Sigma^{q(|x|)} \mid\langle x, w\rangle \in P_{A}\right\}$. In the following we describe an algorithm that on input $x \in A$ computes $w_{\max }$ (the lexicographically largest witness) by a breadth-first search on the tree of prefixes of all potential witnesses. In order to do this we use the set prefix $(\operatorname{Left}(A))=\{\langle x, y\rangle \mid(\exists z)[\langle x, y z\rangle \in \operatorname{Left}(A)]\}$. Each prefix $y$ actually represents the interval of all possible extensions of $y$ to length $q(|x|)$. It is not hard to see that prefix $(\operatorname{Left}(A))$ is many-one equivalent to Left $(A)$ and therefore $\operatorname{prefix}(\operatorname{Left}(A)) \in R_{b}^{p}\left(R_{c t t}^{p}(\mathrm{SPARSE})\right)$.

Using Lemma?? and the representation theorem of Hausdorff stated as Theorem ??, it is easy to see that there exist a sparse set $S$ and sets $C_{i} \in R_{c t t}^{p}(S), 1 \leq i \leq 2 k$, such that prefix $(\operatorname{Left}(A))=\bigcup_{i=1}^{k}\left(C_{2 i-1}-C_{2 i}\right)$ and $C_{1} \supseteq C_{2} \supseteq \ldots \supseteq C_{2 k}$.

Let $f_{i}, 1 \leq i \leq 2 k$, be the conjunctive reduction functions witnessing $C_{i} \in R_{c t t}^{p}(S)$, i.e. $\langle x, y\rangle \in C_{i} \Leftrightarrow f_{i}(\langle x, y\rangle) \subseteq S$.

We first outline an intuitive description of the polynomial-time ${ }^{1}$ decision procedure for A. As stated above, it performs a breadth-first search through the tree of witness prefixes for an input $x$. Let $x$ be an element of $A$, and let $N=\left\{y_{1}, \ldots, y_{t}\right\}$ be a lexicographically ordered set of prefixes (all of the same length) that includes the prefix of $w_{\max }$ of that length. We exploit some crucial properties of the Hausdorff representation of prefix $(\operatorname{Left}(A))$ for the design of a procedure pruning $N$ to a polynomially size-bounded

\footnotetext{
${ }^{1}$ It is implicit in this section that polynomial time and polynomial size always mean polynomial in $|x|$.
} 
set that still includes the prefix of $w_{\max }$. Let $y_{h}$ be the prefix of $w_{\max }$ in $\left\{y_{1}, \ldots, y_{t}\right\}$. Then, letting $d=1$ and $l(0)=1$, it holds that

$$
\left\{\left\langle x, y_{l(d-1)}\right\rangle, \ldots,\left\langle x, y_{h}\right\rangle\right\} \subseteq C_{2 d-1}
$$

Let $r(d)$ be the largest index $r$ such that $\left\{\left\langle x, y_{l(d-1)}\right\rangle, \ldots,\left\langle x, y_{r}\right\rangle\right\} \subseteq C_{2 d-1}$ and let $l(d)$ be the least index $l$ such that $1 \leq l \leq r(d)+1$ and $\left\{\left\langle x, y_{l}\right\rangle, \ldots,\left\langle x, y_{r(d)}\right\rangle\right\} \subseteq C_{2 d}$. Observe that since $\left\{\left\langle x, y_{l(d-1)}\right\rangle, \ldots,\left\langle x, y_{h}\right\rangle\right\} \subseteq C_{2 d-1}$ it follows that $r(d) \geq h$. Similarly, since $\left\{\left\langle x, y_{h+1}\right\rangle, \ldots,\left\langle x, y_{r(d)}\right\rangle\right\} \subseteq C_{2 d}$, it holds that $l(d) \leq h+1$. We consider the following two cases separately.

1. $\left\langle x, y_{h}\right\rangle \notin C_{2 d}$.

Then $l(d)=h+1$ since $y_{h} \notin\left\{y_{l(d)}, \ldots, y_{r(d)}\right\}$, i.e. $l(d)>h$.

2. $\left\langle x, y_{h}\right\rangle \in \mathcal{C}_{2 d}$. (This case is only possible if $d<k$.)

In this case, $y_{h} \in\left\{y_{l(d)}, \ldots, y_{r(d)}\right\}$. Since $\left\{\left\langle x, y_{l(d)}\right\rangle, \ldots,\left\langle x, y_{h}\right\rangle\right\} \subseteq$ prefix $(\operatorname{Left}(A))$ but $\left\{\left\langle x, y_{l(d)}\right\rangle, \ldots,\left\langle x, y_{h}\right\rangle\right\} \subseteq C_{2 d}$, it follows that $\left\{\left\langle x, y_{l(d)}\right\rangle, \ldots,\left\langle x, y_{h}\right\rangle\right\} \subseteq C_{2 d+1}$, and the above analysis can be repeated.

If we could compute the prefixes $y_{l(d)}$ and $y_{r(d)}$ defined above in polynomial time, we could use the above properties in order to design a recursive procedure that collects all the prefixes $y_{l(d)-1}$ found in the recursive calls. This procedure would return a small subset of $N$ containing $y_{h}$. Starting with $N=\{\epsilon\}$, the overall algorithm can use repeatedly such a pruning step at each level of the tree of possible witness prefixes by first expanding all the prefixes $y$ in $N$ to $y 0$ and $y 1$ (thus doubling $N$ ) and then pruning $N$ back to a small subset. In that way, the algorithm finally computes a small subset of $\Sigma^{q(|x|)}$ that contains $w_{\max }$ in case $x \in A$.

Although we cannot explicitly compute the required prefixes $y_{l(d)}$ and $y_{r(d)}$, instead we can compute, given $y_{l(d-1)}$, in polynomial time (polynomially size-bounded) sets $J_{\text {right }}(d)$ and $J_{l e f t}(d)$ of prefixes such that $y_{r(d)} \in J_{r i g h t}(d)$ and $y_{l(d)} \in J_{l e f t}(d)$. This suffices since for each prefix candidate $y \in J_{l e f t}(d)$, the search for $y_{l(d+1)}$ can be done recursively. Since the depth of the recursion is the constant $k$, the resulting sets $J_{l e f t}(d)$ of candidates for $y_{l(d)}$ still have polynomially bounded cardinality.

We now describe the algorithm in detail. It calls a recursive pruning procedure PRUNE which in turn calls two functions SEARCH-RIGHT and SEARCH-LEFT. SEARCHRIGHT is used to search for candidates for $y_{r(d)}$ to the right of previously found candidates for $y_{l(d-1)}$ resulting in a polynomial size-bounded set $J_{r i g h t}(d)$ containing $y_{r(d)}$. SEARCH-LEFT is used to search to the left of the prefixes in $J_{\text {right }}(d)$ to form a polynomial size-bounded set $J_{l e f t}(d)$ containing $y_{l(d)}$. Let $m$ be a polynomial bounding the size of the queries to the sparse set, i.e. $|z| \leq m(n)$ for all $z \in \bigcup\left\{f_{i}(\langle x, y\rangle)|1 \leq i \leq 2 k| x \mid,=\right.$ $n,|y| \leq r(n)\}$, and let $s$ be a polynomial bounding the census of the sparse set $S$.

SEARCH-RIGHT $\left(d, N, y_{l}, x\right)$

(* if $\left\langle x, y_{l}\right\rangle \in C_{2 d-1}$ it returns a set $J \subseteq N=\left\{y_{1}, \ldots, y_{t}\right\}$ that includes the

largest prefix $y_{r} \in N$ such that $\left.\left\{\left\langle x, y_{l}\right\rangle, \ldots,\left\langle x, y_{r}\right\rangle\right\} \subseteq C_{2 d-1}{ }^{*}\right)$

\section{begin}

$$
\begin{aligned}
& J:=\left\{y_{t}\right\} \\
& Q:=\emptyset
\end{aligned}
$$




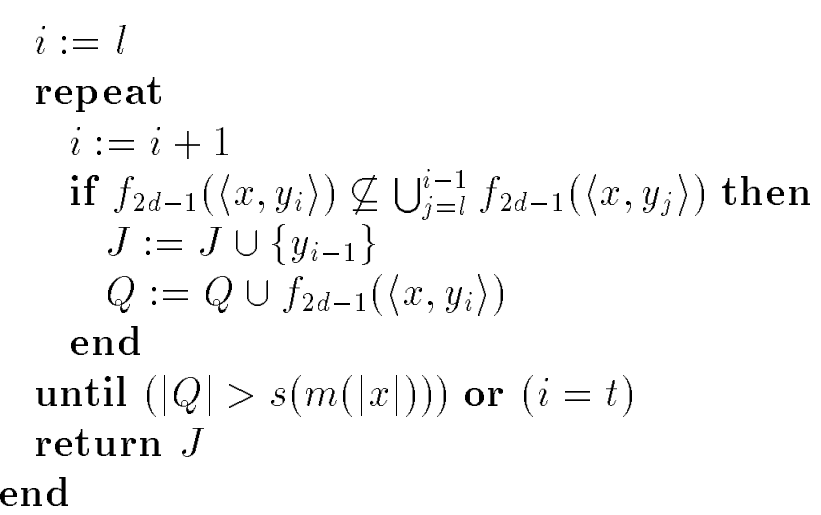

Claim 1 Function SEARCH-RIGHT $\left(d, N, y_{l}, x\right)$, when called with parameter $y_{l}=y_{l(d-1)}$, returns a set $J$ containing $y_{r(d)}$.

Proof of Claim ?? There are two cases. If $r(d)=t$ then $y_{r(d)}$ is clearly in the returned set $J$. Otherwise, since $\left\{\left\langle x, y_{l(d-1)}\right\rangle, \ldots,\left\langle x, y_{r(d)}\right\rangle\right\} \subseteq C_{2 d-1}$ and $\left\langle x, y_{r(d)+1}\right\rangle \notin C_{2 d-1}$, all the queries in the sets $f_{2 d-1}\left(\left\langle x, y_{l(d-1)}\right\rangle\right), \ldots, f_{2 d-1}\left(\left\langle x, y_{r(d)}\right\rangle\right)$ are in $S$ but at least one query $q$ in $f_{2 d-1}\left(\left\langle x, y_{r(d)+1}\right\rangle\right)$ is not in $S$. Therefore $y_{r(d)+1}$ is the smallest prefix $y$ in $N$ such that $y \geq y_{l(d-1)}$ and $q \in f_{d}(\langle x, y\rangle)$, i.e. $y_{r(d)}$ is included in $J$ in some step of the repeat loop since $\left|\bigcup_{j=l(d)}^{r(d)} f_{2 d-1}\left(\left\langle x, y_{j}\right\rangle\right)\right| \leq s(m(|x|))$.

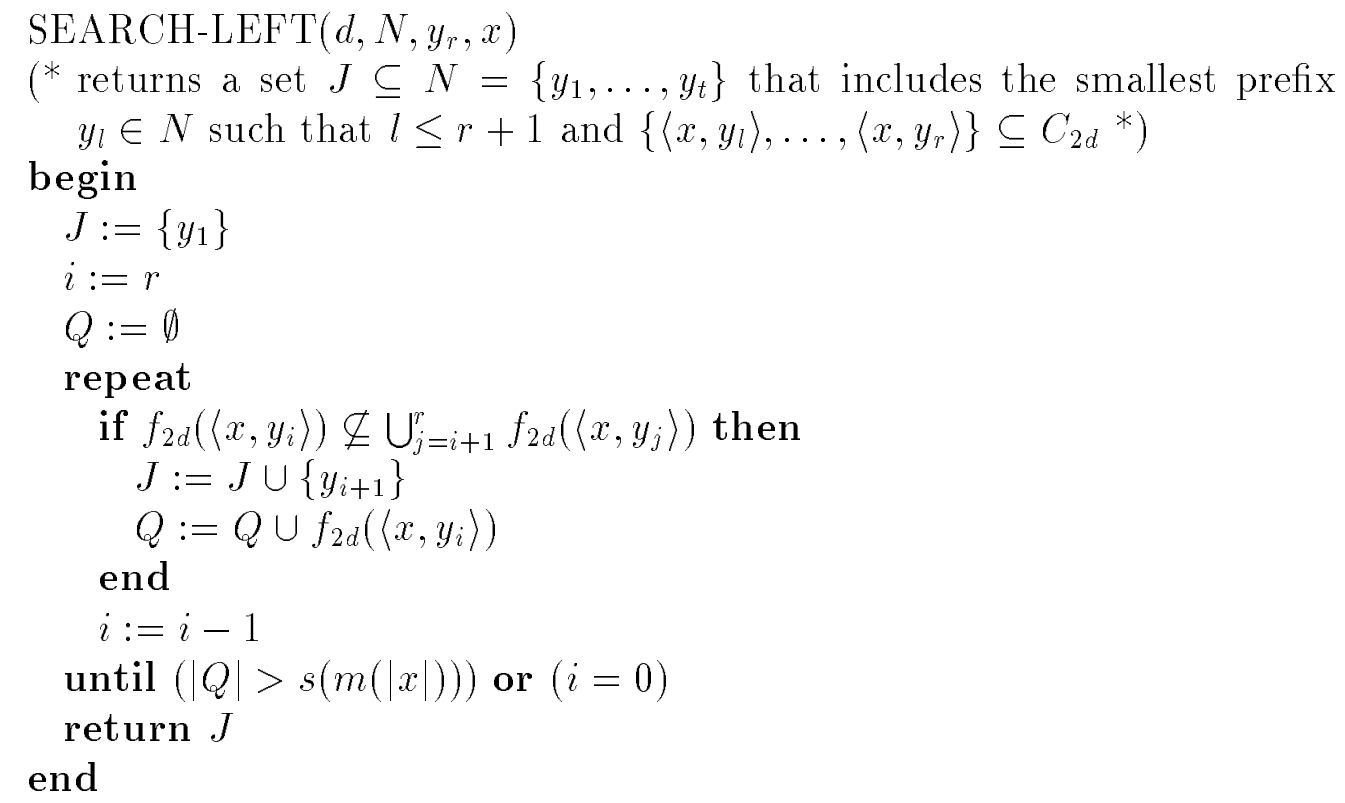

Claim 2 Function SEARCH-LEFT $\left(d, N, y_{r}, x\right)$, when called with parameter $y_{r}=y_{r(d)}$, returns a set $J$ containing $y_{l(d)}$.

Proof of Claim ?? Again, there are two cases. If $l(d)=1$ then $y_{l(d)}$ is clearly in the returned set $J$. Otherwise, since $\left\{\left\langle x, y_{l(d)}\right\rangle, \ldots,\left\langle x, y_{r(d)}\right\rangle\right\} \subseteq C_{2 d}$ and $\left\langle x, y_{l(d)-1}\right\rangle \notin C_{2 d}$, all the queries in the sets $f_{2 d}\left(\left\langle x, y_{l(d)}\right\rangle\right), \ldots, f_{2 d}\left(\left\langle x, y_{r(d)}\right\rangle\right)$ are in $S$ but at least one query $q$ in $f_{2 d}\left(\left\langle x, y_{l(d)-1}\right\rangle\right)$ is not in $S$. Therefore $y_{l(d)-1}$ is the largest prefix $y$ in $N$ such that $y \leq y_{r(d)}$ and $q \in f_{2 d}(\langle x, y\rangle)$, i.e. $y_{l(d)}$ is included in $J$ in some step of the repeat loop since $\left|\bigcup_{j=l(d)}^{r(d)} f_{2 d}\left(\left\langle x, y_{j}\right\rangle\right)\right| \leq s(m(|x|))$. 
$\operatorname{PRUNE}\left(N, J_{l e f t}^{\prime}, d, x\right)$

(* returns a subset of $N=\left\{y_{1}, \ldots, y_{t}\right\}$ that contains the prefix $y_{h}$ of $w_{\max }$ if $y_{h} \in N,\left\langle x, y_{h}\right\rangle \in C_{2 d-1}$ and $\left\{\left\langle x, y_{l}\right\rangle, \ldots,\left\langle x, y_{h}\right\rangle\right\} \subseteq C_{2 d-1}$ for a $y_{l} \in J_{l e f t}^{\prime}$ with $\left.l \leq h^{*}\right)$

\section{begin}

if $d=k+1$ then return $\emptyset$ end

$J_{\text {right }}:=\emptyset$

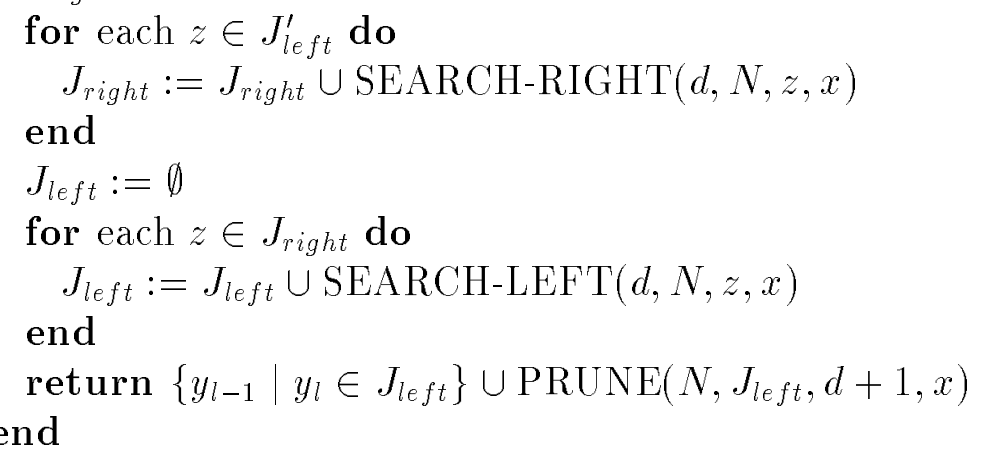

Claim 3 If $y_{h} \in N,\left\langle x, y_{h}\right\rangle \in C_{2 d-1}$, and $y_{l(d-1)} \in J_{l e f t}^{\prime}$ then $\operatorname{PRUNE}\left(N, J_{l e f t}^{\prime}, d, x\right) r e-$ turns a set containing $y_{h}$.

Proof of Claim ?? If $y_{h} \in N$ and $\left\langle x, y_{h}\right\rangle \in C_{2 d-1}$ then $\left\langle x, y_{h}\right\rangle$ is also in the sets $C_{2 d-2}, \ldots, C_{1}$. By the above analysis (since always case 2 happens up to $d-1$ ) it follows that $\left\{\left\langle x, y_{l(d-1)}\right\rangle, \ldots,\left\langle x, y_{h}\right\rangle\right\} \subseteq C_{2 d-1}$. Since $y_{l(d-1)} \in J_{l e f t}^{\prime}$, using Claim ??, $y_{r(d)}$ is included in $J_{\text {right }}$ by the call of SEARCH-RIGHT $\left(d, N, y_{l(d-1)}, x\right)$. Then, using Claim ??, $y_{l(d)}$ is included in $J_{l e f t}$ by the call of SEARCH-LEFT $\left(d, N, y_{r(d)}, x\right)$. Now we can prove by induction that $y_{h}$ is included in the set returned by PRUNE. If $\left\langle x, y_{h}\right\rangle \notin C_{2 d}$ (which must be true in the base case $d=k$ ) then $y_{h}=y_{l(d)-1}$ and $y_{h}$ is included in the set returned by PRUNE. If $\left\langle x, y_{h}\right\rangle \in C_{2 d}$ then $\left\langle x, y_{h}\right\rangle$ is in $C_{2 d+1}$ and we can use the induction hypothesis.

We complete the algorithm with a description of the main program.

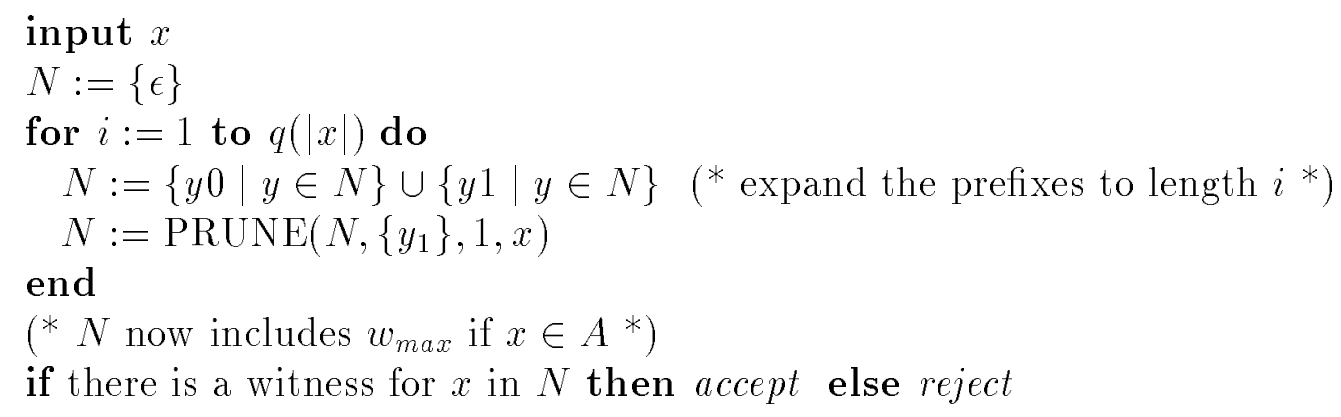

In order to prove the correctness of the algorithm it suffices to observe that it follows from Claim ?? that the prefix $y_{h}$ of $w_{\max }$ is included in the pruned set returned by PRUNE $\left(N,\left\{y_{1}\right\}, 1, x\right)$ provided that $y_{h}$ is in $N$. Also, since the sets returned by SEARCHRIGHT and SEARCH-LEFT are bounded in size by $s(m(|x|))+2$, it follows inductively that the set $J_{l e f t}$ computed by PRUNE at level $d$ is bounded in size by $(s(m(|x|))+2)^{2 d}$. Therefore, since the depth of recursion of function PRUNE is bounded by a constant, the finally returned set being the union of all the $J_{l e f t}$ 's is polynomially bounded in size, and it is easy to see that the algorithm runs in polynomial time. 
We now discuss the application of the above results to the classes UP, FewP, Few, $\mathrm{PP}$, and $\mathrm{C}_{=} \mathrm{P}$.

Theorem 3.5 If UP is contained in $R_{b}^{p}\left(R_{c}^{p}(\mathrm{SPARSE})\right)$ then $\mathrm{UP}=\mathrm{P}$.

Proof We first note that for every set $A \in \mathrm{UP}$ it holds that Left $(A)$ is in UP. Hence, if $\mathrm{UP} \subseteq R_{b}^{p}\left(R_{c}^{p}\right.$ (SPARSE)) then Left $(A)$ is in $R_{b}^{p}\left(R_{c}^{p}\right.$ (SPARSE)), and by Theorem ?? it follows that $A$ is in $\mathrm{P}$.

Theorem 3.6 If FewP is contained in $R_{b}^{p}\left(R_{c}^{p}(\mathrm{SPARSE})\right)$ then $\mathrm{P}=$ Few.

Proof By a similar proof as above it can be inferred that $\mathrm{P}=$ FewP. Since Few $\subseteq \mathrm{P}^{\text {Few }} \mathrm{P}$ $[?]$ it follows that $\mathrm{P}=$ Few.

Theorem 3.7 If $\mathrm{PP}$ is contained in $R_{b}^{p}\left(R_{c t t}^{p}(\mathrm{SPARSE})\right)$ then $\mathrm{P}=\mathrm{PP}$.

Proof Consider the PP-complete set $\{\langle x, m\rangle \mid$ there are at least $m$ satisfying assignments for $x\}$ which has exactly the required properties of left sets. Under the assumption that this set is in $R_{b}^{p}\left(R_{c t t}^{p}(\right.$ SPARSE $\left.)\right)$ we can use the algorithm described in the proof of Theorem ?? to compute in polynomial time a set of numbers that includes the number $\# \operatorname{SAT}(x)$ of satisfying assignments of the formula $x$. Now we can use the result of Cai and Hemachandra [?] and Toda (see [?]) that P $=\mathrm{PP}$ if there is an FP function that computes on input $x$ a set of numbers that includes \#SAT $(x)$.

Theorem 3.8 If $\mathrm{C}_{=} \mathrm{P}$ is contained in $R_{b}^{p}\left(R_{c t t}^{p}(\mathrm{SPARSE})\right)$ then $\mathrm{P}=\mathrm{C}_{=} \mathrm{P}$.

Proof There exist complete sets in $\mathrm{C}_{=} \mathrm{P}$ that are one word decreasing self-reducible [?]. Balcázar has shown that every one word decreasing self-reducible set in $R_{T}^{p}$ (SPARSE) is in $\Sigma_{2}^{p}$ [?]. Therefore it follows from the assumption of the theorem that $\mathrm{C}=\mathrm{P} \subseteq \Sigma_{2}^{p}$. Furthermore, since coNP $\subseteq \mathrm{C}_{=} \mathrm{P}$, if $\mathrm{C}_{=} \mathrm{P} \subseteq R_{b}^{p}\left(R_{c t t}^{p}(\mathrm{SPARSE})\right)$ then also NP $\subseteq$ $R_{b}^{p}\left(R_{c t t}^{p}(\mathrm{SPARSE})\right)$, and it follows from Theorem ?? that $\mathrm{P}=\Sigma_{2}^{p}$.

Theorem ?? could also be proved in the same way as Theorem ??

\section{Bounded truth-tables on randomized reductions to sparse sets}

In this section we consider randomized reductions to sparse sets. Randomized reductions were introduced by Adleman and Manders [?] and have played an important role in complexity theory. We show that NP cannot have sparse hard sets under certain randomized reductions unless NP $=\mathrm{RP}$. Namely, we show that if NP reduces via a bounded truth-table reduction to a set that reduces via a co-rp reduction to a sparse set then $\mathrm{NP}=\mathrm{RP}$.

Definition 4.1 [?] $A \leq_{m}^{r p} B$ if there exist a polynomial time function $f$ and a polynomial q such that 


$$
\begin{aligned}
& x \in A \Rightarrow \operatorname{Prob}_{w \in \Sigma^{q}(|x|)}[f(\langle x, w\rangle) \in B] \geq 3 / 4 \\
& x \notin A \Rightarrow \operatorname{Prob}_{w \in \Sigma^{q}(|x|)}[f(\langle x, w\rangle) \notin B]=1
\end{aligned}
$$

Similarly, $A \leq_{m}^{c o-r p} B$ if there exist a polynomial time function $f$ and a polynomial q such that

$$
\begin{aligned}
& x \in A \Rightarrow \operatorname{Prob}_{w \in \Sigma q(|x|)}[f(\langle x, w\rangle) \in B]=1 \\
& x \notin A \Rightarrow \operatorname{Prob}_{w \in \Sigma q(|x|)}[f(\langle x, w\rangle) \notin B] \geq 3 / 4
\end{aligned}
$$

The string $w$ is chosen uniformly at random from the set $\Sigma^{q(|x|)}$.

We first show that if NP $\subseteq R_{m}^{c o-r p}\left(R_{c t t}^{p}(\right.$ SPARSE)) then NP $=R P$ (this result is independently due to D. Ranjan and P. Rohatgi [?]). Then we extend this to the result that $\mathrm{NP} \subseteq R_{b}^{p}\left(R_{m}^{c o-r p}\left(R_{c t t}^{p}(\mathrm{SPARSE})\right)\right)$ implies NP $=\mathrm{RP}$. We need the following folklore result on amplification for randomized reductions.

Lemma 4.2 If $A \leq_{m}^{c o-r p} B$ then for every polynomial $p$ there exist a co-rp reduction function $f$ from $A$ to $A N D_{\omega}(B)=\left\{\left\langle x_{1}, \ldots, x_{i}\right\rangle \mid x_{j} \in B\right.$ for each $\left.j, 1 \leq j \leq i\right\}$ and $a$ polynomial q such that

$$
\begin{aligned}
& x \in A \Rightarrow \operatorname{Prob}_{w \in \Sigma q(|x|)}\left[f(\langle x, w\rangle) \in A N D_{\omega}(B)\right]=1 \\
& x \notin A \Rightarrow \operatorname{Prob}_{w \in \Sigma q(|x|)}\left[f(\langle x, w\rangle) \notin A N D_{\omega}(B)\right] \geq 1-2^{-p(|x|) .}
\end{aligned}
$$

Fact 4.3 For every set $B, A N D_{\omega}(B) \in R_{c t t}^{p}(B)$.

Fact ?? shows that if a set $B$ conjunctively reduces in polynomial time to a sparse set $S$ then $\operatorname{AND}_{\omega}(B)$ also conjunctively reduces to $S$. The following lemma is an easy consequence of Lemma ?? and Fact ??

Lemma 4.4 If $A \in R_{m}^{c o-r p}\left(R_{c t t}^{p}(\mathrm{SPARSE})\right)$ then for every polynomial p there exist a sparse set $S$, an FP function $f$, and a polynomial q such that

$$
\begin{aligned}
& x \in A \Rightarrow \operatorname{Prob}_{w \in \Sigma q|x|)}[f(\langle x, w\rangle) \subseteq S]=1 \\
& x \notin A \Rightarrow \operatorname{Prob}_{w \in \Sigma q|x|)}[f(\langle x, w\rangle) \nsubseteq S] \geq\left(1-2^{-p(|x|)}\right)
\end{aligned}
$$

The following result has been independently obtained by D. Ranjan and P. Rohatgi $[?])$.

Theorem 4.5 If NP $\subseteq R_{m}^{c o-r p}\left(R_{c t t}^{p}(\mathrm{SPARSE})\right)$ then $\mathrm{NP}=\mathrm{RP}$.

Proof Let $A$ be an NP-complete set such that $A \in R_{m}^{c o-r p}\left(R_{c t t}^{p}\right.$ (SPARSE)). As in the proof of Theorem ?? let $r$ be a polynomial and let $P_{A}$ be a polynomial-time set such that $A=\left\{x \mid \exists w \in \Sigma^{r(|x|)}:\langle x, w\rangle \in P_{A}\right\}$ and Left $(A)=\left\{\langle x, w\rangle \mid x \in A \wedge w \in \Sigma^{r(|x|)} \wedge w \leq\right.$ $\left.w_{\max }\right\}$, where $w_{\max }=\max \left\{w \in \Sigma^{r(|x|)} \mid\langle x, w\rangle \in P_{A}\right\}$.

We describe a randomized polynomial time algorithm that computes on input $x$ in $A$ with high probability the lexicographically largest witness $w_{\max }$ by a breadth-first search on the tree of possible witness prefixes. In order to do this we again use the set $\operatorname{prefix}(\operatorname{Left}(A))=\{\langle x, y\rangle \mid \exists z:\langle x, y z\rangle \in \operatorname{Left}(A)\}$ which is in $R_{m}^{c o-r p}\left(R_{c t t}^{p}(\right.$ SPARSE) $)$ since it is many-one equivalent to $\operatorname{Left}(A)$.

Let $p$ be a polynomial such that for all $n,\left(1-2^{-p(n)}\right)^{r(n)} \geq 3 / 4$. By Lemma ?? there exist a sparse set $S$, an FP function $f$ and a polynomial $q$ such that 


$$
\begin{aligned}
& \langle x, y\rangle \in \operatorname{prefix}(\operatorname{Left}(A)) \Rightarrow \operatorname{Prob}_{w \in \Sigma q(|x|)}[f(\langle x, y, w\rangle) \subseteq S]=1 \\
& \langle x, y\rangle \notin \operatorname{prefix}(\operatorname{Left}(A)) \Rightarrow \operatorname{Prob}_{w \in \Sigma q(|x|)}[f(\langle x, y, w\rangle) \nsubseteq S] \geq 1-2^{-p(|x|)}
\end{aligned}
$$

Let $m$ be a polynomial bounding the size of the queries to the sparse set, i.e. $|z| \leq m(n)$ for all $z \in \bigcup\{f(\langle x, y, w\rangle)|| x|=n| y,|\leq r(n)| w \mid,=q(n)\}$, and let $s$ be a polynomial bounding the census of the sparse set $S$.

We first describe the randomized algorithm for testing membership in $A$ and then prove its correctness.

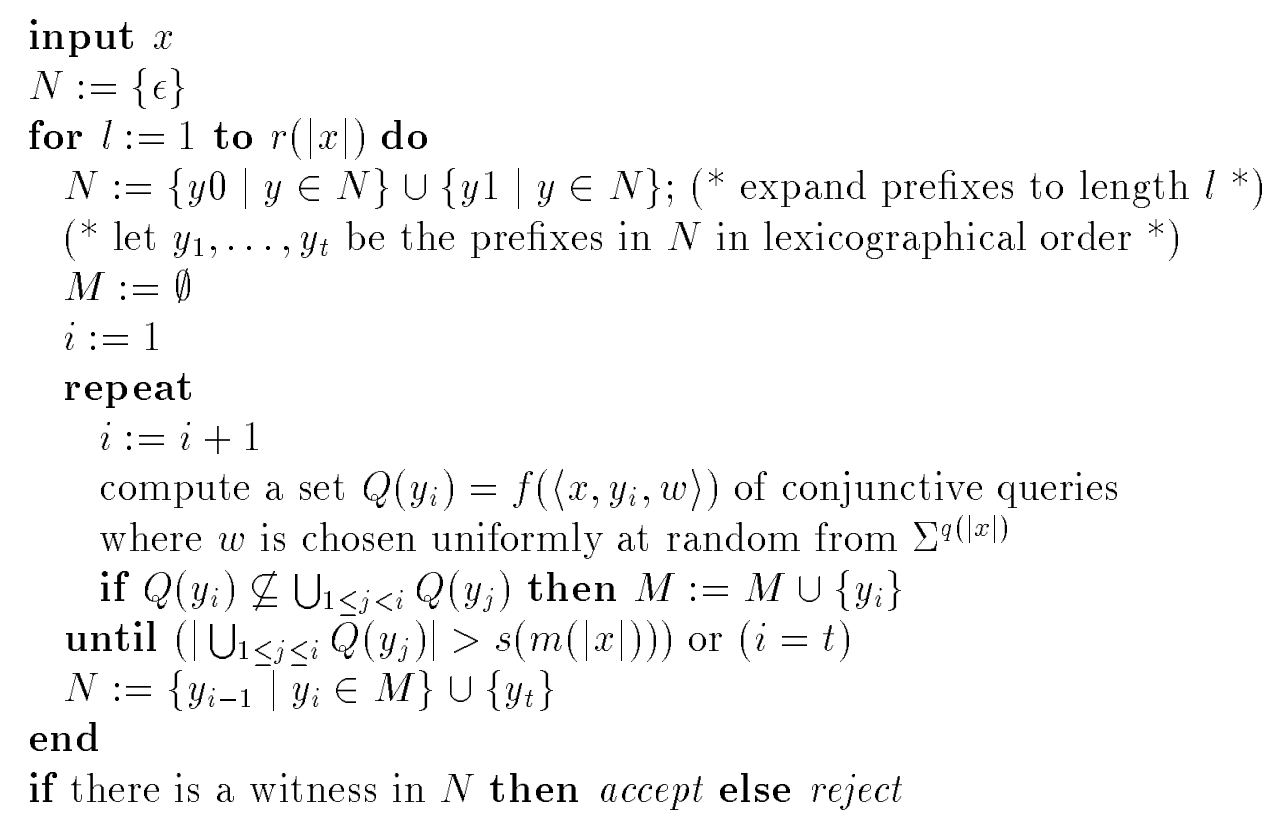

It is clear that the above algorithm runs in polynomial time since the set $N$ contains at most $2(s(m(|x|))+2)$ prefixes at any stage of the loop. In order to prove that it is an RP algorithm for $A$ we need to show that if $x \in A$ then the algorithm accepts $x$ with high probability, and if $x \notin A$ then the algorithm always rejects. The latter is obvious from the fact that the algorithm accepts only if it finds a witness. It remains to show that if $x \in A$ then the algorithm finds $w_{\max }$ with probability at least $3 / 4$.

We show that if $N=\left\{y_{1}, \ldots, y_{t}\right\}$ contains a prefix of $w_{\max }$ (call it $y_{h}$; we assume that $h<t$ since $y_{t}$ is always included in the pruned set) then with probability at least $1-2^{-p(|x|)}$ the prefix $y_{h}$ is included in $N$ after the repeat loop. In order to see this we observe the following.

1. For every $w \in \Sigma^{q(|x|)}$ and $i, 1 \leq i \leq h$, it holds that $f\left(\left\langle x, y_{i}, w\right\rangle\right) \subseteq S$. This follows from the fact that $\left\langle x, y_{i}\right\rangle \in \operatorname{prefix}(\operatorname{Left}(A))$ for $1 \leq i \leq h$.

2. Since $\left\langle x, y_{h+1}\right\rangle \notin \operatorname{prefix}(\operatorname{Left}(A))$, it holds that $\left.\operatorname{Prob}_{w \in \Sigma} q|x|\right)\left[f\left(\left\langle x, y_{h+1}, w\right\rangle\right) \nsubseteq S\right] \geq$ $\left(1-2^{-p(|x|)}\right)$.

It follows that $\left|\bigcup_{1 \leq j \leq h} Q\left(y_{j}\right)\right| \leq s(m(|x|))$ and with probability at least $1-2^{-p(|x|)}$ it holds that $Q\left(y_{h+1}\right) \not \subset \bigcup_{1 \leq j \leq h} Q\left(y_{j}\right)$. Hence $N$ includes $y_{h}$ with probability at least $1-2^{-p(|x|)}$ at the end of the repeat loop.

Since the outer for-loop has $r(|x|)$ iterations, and since at the beginning $N=\{\epsilon\}$ contains a prefix of $w_{\max }$, it follows that with probability at least $\left(1-2^{-p(|x|)}\right)^{r(|x|)}$ the algorithm finds $w_{\max }$. By choice of $p$ this probability is more than $3 / 4$. 
We state the next theorem without proof as it can be proved exactly as Theorem ??

Theorem 4.6 If NP $\subseteq R_{m}^{r p}\left(R_{d t t}^{p}(\right.$ co-SPARSE $\left.)\right)$ then $\mathrm{NP}=\mathrm{RP}$.

We now extend the above results to prove that if NP $\subseteq R_{b}^{p}\left(R_{m}^{c o-r p}\left(R_{c t t}^{p}(\mathrm{SPARSE})\right)\right)$ then NP $=$ RP. We first show that the class $R_{m}^{c o-r p}\left(R_{c t t}^{p}(\mathrm{SPARSE})\right)$ is a set ring so that we can assume the existence of a Hausdorff representation for any set in $R_{b}^{p}\left(R_{m}^{c o-r p}\left(R_{c t t}^{p}(\mathrm{SPARSE})\right)\right)$.

\section{Lemma $4.7 \quad$ 1. $R_{m}^{c o-r p}\left(R_{c t t}^{p}(\operatorname{SPARSE})\right)=R_{m}^{c o-r p}\left(R_{c t t}^{p}(\right.$ TALLY $\left.)\right)$}

D. $R_{m}^{r p}\left(R_{d t t}^{p}(\right.$ co-SPARSE $\left.)\right)=R_{m}^{r p}\left(R_{d t t}^{p}(T A L L Y)\right)$

Proof Follows from the facts that $R_{d t t}($ co-SPARSE $)=R_{d t t}($ TALLY $)$ and that $R_{c t t}^{p}(\mathrm{SPARSE})=R_{c t t}^{p}(\mathrm{TALLY})$.

Lemma $4.8 R_{m}^{c o-r p}\left(R_{c t t}^{p}(\right.$ SPARSE $\left.)\right)$ is a set ring.

Proof Since $R_{m}^{c o-r p}\left(R_{c t t}^{p}(\mathrm{SPARSE})\right)=R_{m}^{c o-r p}\left(R_{c t t}^{p}(\right.$ TALLY $\left.)\right)$, it is enough to show that $R_{m}^{c o-r p}\left(R_{c t t}^{p}(\right.$ TALLY $\left.)\right)$ is a set ring. Assume that $A \leq_{m}^{c o-r p} A_{1}$ and $B \leq_{m}^{c o-r p} B_{1}$, where $A_{1}$ and $B_{1}$ are in $R_{c t t}^{p}($ TALLY $)$. We define two sets $C$ and $D$ which are readily seen to be in $R_{c t t}^{p}($ TALLY $)$ since $A_{1}$ and $B_{1}$ are tally sets and since $R_{b d}^{p}\left(R_{c t t}^{p}(\right.$ TALLY $\left.)\right)=R_{c t t}^{p}($ TALLY $)$ and $R_{b c}^{p}\left(R_{c t t}^{p}(\right.$ TALLY $\left.)\right)=R_{c t t}^{p}($ TALLY $)$.

$$
\begin{aligned}
& C=\left\{\langle a, b\rangle \mid a \in A_{1} \text { or } b \in B_{1}\right\} \\
& D=\left\{\langle a, b\rangle \mid a \in A_{1} \text { and } b \in B_{1}\right\} .
\end{aligned}
$$

Let $f$ and $g$ be co-rp reduction functions witnessing $A \leq_{m}^{c o-r p} A_{1}$ and $B \leq_{m}^{c o-r p} B_{1}$, respectively. We can assume that there is a uniform polynomial $q$ corresponding to both reduction functions such that

$$
\begin{aligned}
& x \in A \Rightarrow \operatorname{Prob}_{w \in \Sigma q(|x|)}\left[f(\langle x, w\rangle) \in A_{1}\right]=1 \\
& x \notin A \Rightarrow \operatorname{Prob}_{w \in \Sigma q(|x|)}\left[f(\langle x, w\rangle) \notin A_{1}\right] \geq 3 / 4
\end{aligned}
$$

and

$$
\begin{aligned}
& x \in B \Rightarrow \operatorname{Prob}_{w \in \Sigma q(|x|)}\left[g(\langle x, w\rangle) \in B_{1}\right]=1 \\
& x \notin B \Rightarrow \operatorname{Prob}_{w \in \Sigma q(|x|)}\left[g(\langle x, w\rangle) \notin B_{1}\right] \geq 3 / 4
\end{aligned}
$$

We define a reduction function $h$ combining $f$ and $g$ as follows. For $w_{1}, w_{2} \in \Sigma^{q(|x|)}$, $h\left(\left\langle x, w_{1} w_{2}\right\rangle\right)=\left\langle f\left(\left\langle x, w_{1}\right\rangle\right), g\left(\left\langle x, w_{2}\right\rangle\right)\right\rangle$. Then we have

$$
\begin{aligned}
& x \in A \cup B \Rightarrow \operatorname{Prob}_{w \in \Sigma^{2 q}(|x|)}[h(\langle x, w\rangle) \in C]=1 \\
& x \notin A \cup B \Rightarrow \operatorname{Prob}_{w \in \Sigma^{2 q}(|x|)}[h(\langle x, w\rangle) \notin C] \geq(3 / 4)^{2}
\end{aligned}
$$

Note that the probability $(3 / 4)^{2}$ can be amplified using Lemma?? to $3 / 4$ as required. Similarly we have

$$
x \in A \cap B \Rightarrow \operatorname{Prob}_{w \in \Sigma^{2 q(|x|)}}[h(\langle x, w\rangle) \in D]=1
$$




$$
x \notin A \cap B \Rightarrow \operatorname{Prob}_{w \in \Sigma^{2 q(|x|)}}[h(\langle x, w\rangle) \notin D] \geq 1-(1 / 4)^{2} .
$$

Hence $A \cap B$ and $A \cup B$ co-rp reduce to sets in $R_{c t t}^{p}$ (TALLY), i.e. $R_{m}^{c o-r p}\left(R_{c t t}^{p}(\right.$ SPARSE $\left.)\right)$ is a set ring.

We are now ready to prove the main result of this section.

Theorem 4.9 If $A$ is in NP such that Left $(A) \in R_{b}^{p}\left(R_{m}^{c o-r p}\left(R_{c t t}^{p}(\mathrm{SPARSE})\right)\right)$ then $A \in$ RP.

Proof The proof is quite similar to that of Theorem ??. The essential difference is that the procedures corresponding to SEARCH-LEFT and SEARCH-RIGHT in the proof of Theorem ?? will now be randomized algorithms (similar to the pruning part used in the algorithm in the proof of Theorem ??).

Let $q$ be a polynomial and let $P_{A}$ be a polynomial-time set such that $A=\{x \mid \exists w \in$ $\left.\Sigma^{r(|x|)}:\langle x, w\rangle \in P_{A}\right\}$ and $\operatorname{Left}(A)=\left\{\langle x, w\rangle \mid x \in A \wedge w \in \Sigma^{r(|x|)} \wedge w \leq w_{\max }\right\}$, where $w_{\max }=\max \left\{w \in \Sigma^{r(|x|)} \mid\langle x, w\rangle \in P_{A}\right\}$. We describe an RP-algorithm that on input $x \in A$ computes with high probability $w_{\max }$ by a breadth-first search. By the hypothesis of the theorem we can assume that the set prefix $(\operatorname{Left}(A))=\{\langle x, y\rangle \mid$ $\exists z:\langle x, y z\rangle \in \operatorname{Left}(A)\}$ is in $R_{b}^{p}\left(R_{m}^{c 0^{-r p}}\left(R_{c t t}^{p}(\mathrm{SPARSE})\right)\right)$. Since $R_{m}^{c 0^{-r p}}\left(R_{c t t}^{p}(\mathrm{SPARSE})\right)$ is closed under join and polynomial time many-one reductions, it follows from Fact ?? that $B C\left(R_{m}^{c o^{-r} p}\left(R_{c t t}^{p}(\mathrm{SPARSE})\right)=R_{b}^{p}\left(R_{m}^{c c^{-r p}}\left(R_{c t t}^{p}(\mathrm{SPARSE})\right)\right)\right.$. From Lemma ?? and Theorem ??, it follows that there exist a sparse set $S$ and sets $C_{i} \in R_{m}^{c o-r p}\left(R_{c t t}^{p}(S)\right)$ such that prefix $(\operatorname{Left}(A))=\bigcup_{i=1}^{k}\left(C_{2 i-1}-C_{2 i}\right)$ and $C_{1} \supseteq C_{2} \supseteq \ldots \supseteq C_{2 k}$.

Let $p$ be a polynomial such that for all $n,\left(1-2^{-p(n)}\right)^{r(n) \cdot 2 k} \geq 3 / 4$. Then by Lemma ?? we can assume that there exist co-rp reduction functions $f_{i}$ witnessing $C_{i} \in R_{m}^{c o-r p}\left(R_{c t t}^{p}(S)\right), 1 \leq i \leq 2 k$, and a polynomial $q$ such that the following holds

$$
\begin{aligned}
& \langle x, y\rangle \in C_{i} \Rightarrow \operatorname{Prob}_{w \in \Sigma q(|x|)}\left[f_{i}(\langle x, y, w\rangle) \subseteq S\right]=1 \\
& \langle x, y\rangle \notin C_{i} \Rightarrow \operatorname{Prob}_{w \in \Sigma q(|x|)}\left[f_{i}(\langle x, y, w\rangle) \nsubseteq S\right] \geq\left(1-2^{-p(|x|)}\right)
\end{aligned}
$$

The indices $l(d)$ and $r(d), 1 \leq d \leq k$, are defined in the same way as in Theorem ??. Let $y_{h}$ be the prefix of $w_{\max }$ in $\left\{y_{1}, \ldots, y_{t}\right\}$. As in Theorem ??, we cannot compute the required prefixes $y_{l(d)}$ and $y_{r(d)}$. Instead we design a randomized algorithm that, given $y_{l(d-1)}$, computes in polynomial time (polynomially size-bounded) sets $J_{\text {right }}(d)$ and $J_{l e f t}(d)$ of prefixes such that with high probability $y_{r(d)} \in J_{\text {right }}(d)$ and $y_{l(d)} \in J_{l e f t}(d)$. We now describe the algorithm in detail. It calls a recursive pruning procedure PRUNE which in turn calls two functions RANDOM-SEARCH-RIGHT and RANDOM-SEARCHLEFT. RANDOM-SEARCH-RIGHT is used to search for candidates for $y_{r(d)}$ to the right of previously found candidates for $y_{l(d-1)}$ resulting in a polynomially bounded set $J_{\text {right }}(d)$ containing $y_{r(d)}$ with high probability. RANDOM-SEARCH-LEFT is used to search to the left of the prefixes in $J_{\text {right }}(d)$ to form a polynomial size-bounded set $J_{\text {left }}(d)$ containing $y_{l(d)}$ with high probability.

Let $m$ be a polynomial bounding the size of the queries to the sparse set, i.e. $|z| \leq m(n)$ for all $z \in \bigcup\left\{f_{i}(\langle x, y, w\rangle)|1 \leq i \leq 2 k| x,|=n| y,|\leq r(|x|)| w \mid,=q(|x|)\right\}$, and let $s$ be a polynomial bounding the census of the sparse set $S$. 
RANDOM-SEARCH-RIGHT $\left(d, N, y_{l}, x\right)$

(* if $\left\langle x, y_{l}\right\rangle \in C_{2 d-1}$ it returns a set $J \subseteq N=\left\{y_{1}, \ldots, y_{t}\right\}$ that includes with high probability the largest prefix $y_{r} \in N$ such that $\left.\left\{\left\langle x, y_{l}\right\rangle, \ldots,\left\langle x, y_{r}\right\rangle\right\} \subseteq C_{2 d-1} *\right)$

\section{begin}

$$
\begin{aligned}
& J:=\left\{y_{t}\right\} \\
& Q:=\emptyset \\
& i:=l
\end{aligned}
$$

repeat

$i:=i+1$

compute a set $Q\left(y_{i}\right)=f_{2 d-1}\left(\left\langle x, y_{i}, w\right\rangle\right)$ of conjunctive queries where $w$ is chosen uniformly at random from $\Sigma^{q(|x|)}$

if $Q\left(y_{i}\right) \nsubseteq \bigcup_{j=l}^{i-1} Q\left(y_{j}\right)$ then

$J:=J \cup\left\{y_{i-1}\right\}$

$Q:=Q \cup Q\left(y_{i}\right)$

end

until $(|Q|>s(m(|x|)))$ or $(i=t)$

return $J$

end

Claim 4 Function RANDOM-SEARCH-RIGHT $\left(d, N, y_{l}, x\right)$, when called with parameter $y_{l}=y_{l(d-1)}$, returns a set $J$ that with probability at least $1-2^{-p(|x|)}$ contains $y_{r(d)}$.

Proof of Claim ?? We first note that if $r(d)=t$ then $y_{r(d)}$ is always included in $J$. Otherwise observe the following.

1. For every $w \in \Sigma^{q(|x|)}$ and $i, l(d-1) \leq i \leq r(d)$, it holds that $f_{2 d-1}\left(\left\langle x, y_{i}, w\right\rangle\right) \subseteq S$. This follows from the fact that $\left\langle x, y_{i}\right\rangle \in C_{2 d-1}$ for all $i, 1 \leq i \leq r(d)$.

2. Since $\left\langle x, y_{r(d)+1}\right\rangle \notin C_{2 d-1}$ it holds that $\operatorname{Prob}_{w \in \Sigma q(|x|)}\left[f_{2 d-1}\left(\left\langle x, y_{r(d)+1}, w\right\rangle\right) \nsubseteq S\right] \geq$ $1-2^{-p(|x|)}$.

Therefore, since $\left|\bigcup_{j=l(d-1)}^{r(d)} Q\left(y_{j}\right)\right| \leq s(m(|x|))$, the repeat loop includes $y_{r(d)}$ in $J$ with probability at least $1-2^{-p(|x|)}$.

RANDOM-SEARCH-LEFT $\left(d, N, y_{r}, x\right)$

( ${ }^{*}$ returns a set $J \subseteq N=\left\{y_{1}, \ldots, y_{t}\right\}$ that includes with high probability the smallest prefix $y_{l} \in N$ such that $\left.\left\{\left\langle x, y_{l}\right\rangle, \ldots,\left\langle x, y_{r}\right\rangle\right\} \subseteq C_{2 d}{ }^{*}\right)$

begin

$$
\begin{aligned}
& J:=\left\{y_{1}\right\} \\
& i:=r \\
& Q:=\emptyset \\
& \text { repeat }
\end{aligned}
$$

compute a set $Q\left(y_{i}\right)=f_{2 d}\left(\left\langle x, y_{i}, w\right\rangle\right)$ of conjunctive queries where $w$ is chosen uniformly at random from $\Sigma^{q(|x|)}$

if $Q\left(y_{i}\right) \nsubseteq \bigcup_{j=i+1}^{r} Q\left(y_{j}\right)$ then

$J:=J \cup\left\{y_{i+1}\right\}$

$Q:=Q \cup Q\left(y_{i}\right)$

end 
$i:=i-1$

until $(|Q|>s(m(|x|)))$ or $(i=1)$

return $J$

end

Claim 5 Function RANDOM-SEARCH-LEFT $\left(d, N, y_{r}, x\right)$, when called with parameter $y_{r}=y_{r(d)}$, returns a set $J$ containing $y_{l(d)}$ with probability at least $1-2^{-p(|x|)}$.

Proof of Claim ?? There are two cases again. If $l(d)=1$ then $y_{l(d)}$ is clearly in the returned set $J$. Otherwise we have

1. Since $\left\{\left\langle x, y_{l(d)}\right\rangle, \ldots,\left\langle x, y_{r(d)}\right\rangle\right\} \subseteq C_{2 d}$ and $\left\langle x, y_{l(d)-1}\right\rangle \notin C_{2 d}$, it holds for every $w \in$ $\Sigma^{q(|x|)}$ and for every $i, l(d) \leq i \leq r(d)$, that $f_{2 d}\left(\left\langle x, y_{i}, w\right\rangle\right) \subseteq S$.

2. $\operatorname{Prob}_{w \in \Sigma^{q(|x|)}}\left[f_{2 d}\left(\left\langle x, y_{l(d)-1}, w\right\rangle\right) \nsubseteq S\right] \geq\left(1-2^{-p(|x|)}\right)$.

Therefore, since $\left|\bigcup_{j=l(d)}^{r(d)} Q\left(y_{j}\right)\right| \leq s(m(|x|)), y_{l(d)}$ is included in $J$ with probability at least $1-2^{-p(|x|)}$ in some step of the repeat loop. The claim follows.

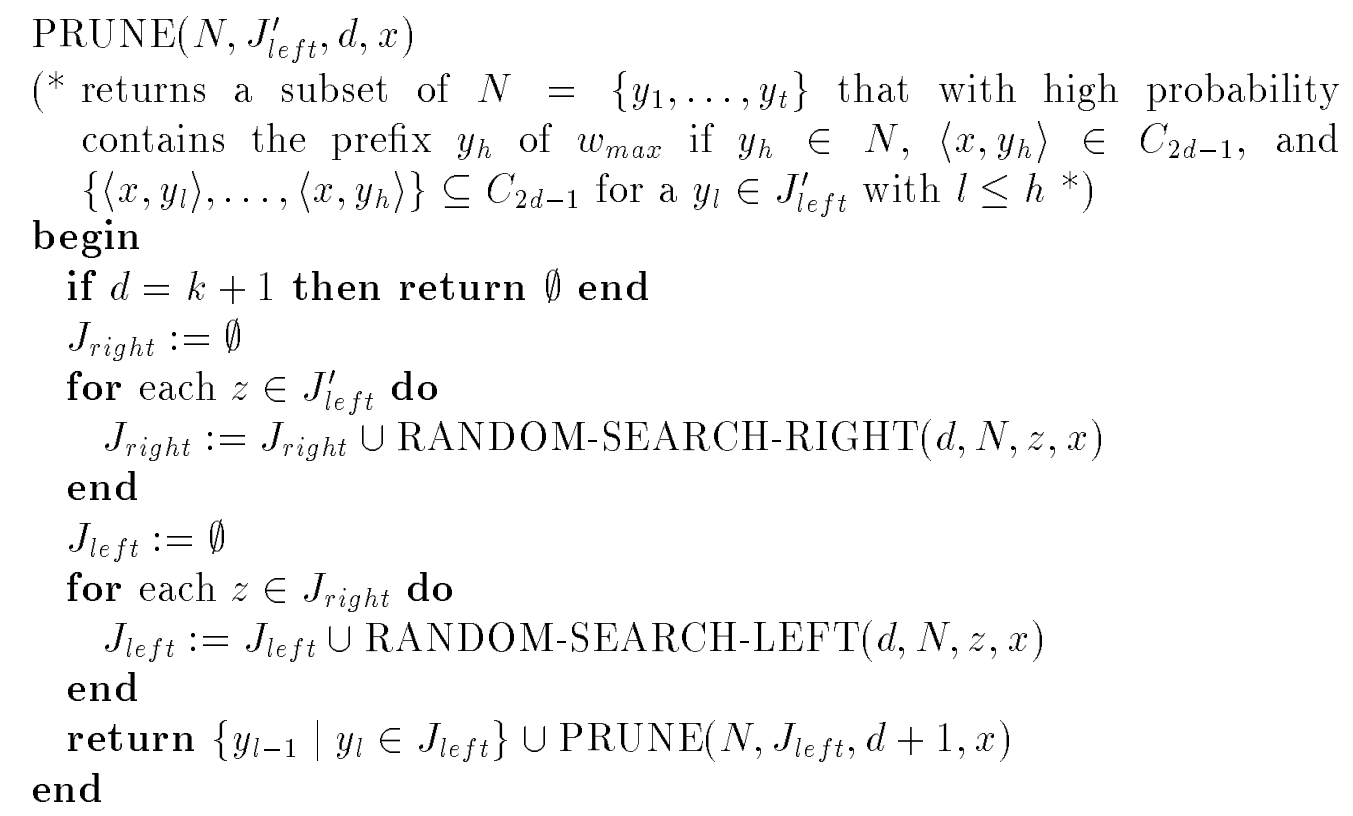

The next claim follows from Claim ?? and Claim ?? and is similarly proved as Claim ?? in Theorem ??.

Claim 6 If $y_{h} \in N,\left\langle x, y_{h}\right\rangle \in C_{2 d-1}$, and $y_{l(d-1)} \in J_{\text {left }}^{\prime}$ then $\operatorname{PRUNE}\left(N, J_{\text {left }}^{\prime}, d, x\right)$ returns a set containing $y_{h}$ with probability at least $\left(1-2^{-p(|x|)}\right)^{2 k}$.

We complete the algorithm with a description of the main program.

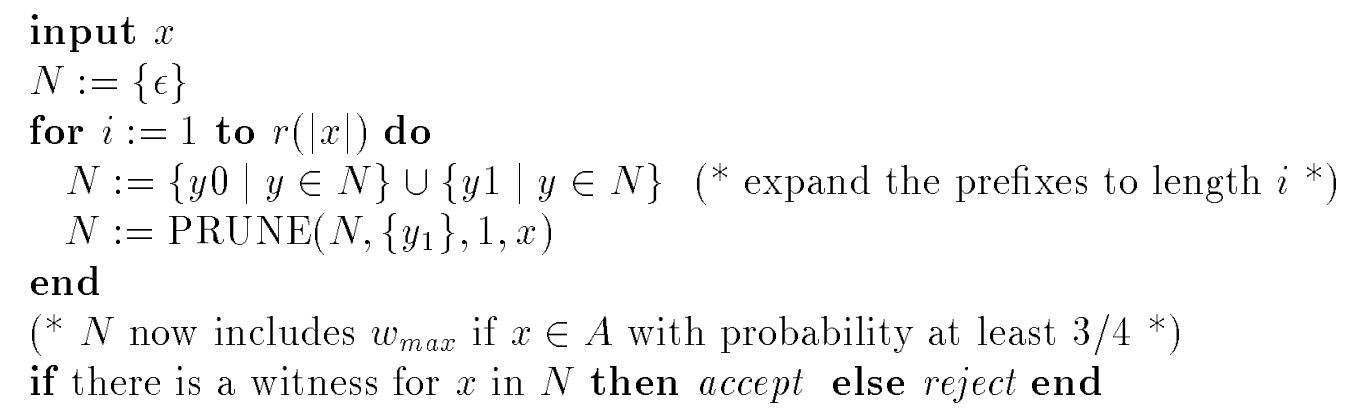


We first note that an input $x \notin A$ is rejected with probability 1 since no witness can be found. In order to prove the correctness of the algorithm it suffices to observe that Claim ?? implies that with probability at least $\left(1-2^{-p(|x|)}\right)^{2 k}$ the prefix $y_{h}$ of $w_{\max }$ is included in the pruned set returned by $\operatorname{PRUNE}\left(N,\left\{y_{1}\right\}, 1, x\right)$ provided that $y_{h}$ is in $N$. Hence, after exiting the for-loop in the main program, $N$ includes $w_{\max }$ with probability at least $\left(\left(1-2^{-p(|x|)}\right)^{2 k}\right)^{r(|x|)}$ (which is more than $3 / 4$ by choice of $p$ ). It is easy to see that the algorithm runs in polynomial time.

The proof of the following theorem is analogous to the proof of Theorem ??

Theorem 4.10 If UP is contained in $R_{b}^{p}\left(R_{m}^{c o-r p}\left(R_{c}^{p}(\mathrm{SPARSE})\right)\right)$ then $\mathrm{UP} \subseteq \mathrm{RP}$.

Proof Since for every set $A \in \mathrm{UP}$ it holds that Left $(A)$ is in UP and since UP $\subseteq$ $R_{b}^{p}\left(R_{m}^{c o-r p}\left(R_{c}^{p}(\mathrm{SPARSE})\right)\right)$ it follows that $\operatorname{Left}(A)$ is in $R_{b}^{p}\left(R_{m}^{c o-r p}\left(R_{c}^{p}(\mathrm{SPARSE})\right)\right)$, and therefore by Theorem ?? $A$ is in RP.

\section{Promise problems and randomized reductions to sparse sets}

We show in this section that it is enough to assume that some solution of the promise problem (1SAT, SAT) is reducible to a sparse set via the randomized reduction considered in Theorem ?? to get the conclusion NP $=\mathrm{RP}$. We first give the definition of promise problems and state its relation to randomized reductions.

Definition 5.1 [?] A promise problem is a pair of sets $(Q, R)$. A set $L$ is called a solution to the promise problem $(Q, R)$ if $(\forall x)[x \in Q \Rightarrow(x \in L \Leftrightarrow x \in R)]$.

Let 1SAT denote the set of formulas with at most one satisfying assignment. Observe that a solution of the promise problem (1SAT, SAT) has to agree with SAT in the formulas having a unique satisfying assignment as well as in the unsatisfiable formulas. The well known result of Valiant and Vazirani showing the NP-hardness of USAT under (a weaker version of) randomized reductions [?] has the following implication for the promise problem (1SAT, SAT).

Theorem 5.2 [?] If the promise problem (1SAT, SAT) has a solution in $\mathrm{RP}$ then $\mathrm{NP}=$ RP.

We now prove the generalization of Theorem ??.

Theorem 5.3 If the promise problem (1SAT, SAT) has a solution in the reduction class $R_{b}^{p}\left(R_{m}^{c o-r p}\left(R_{c}^{p}(\mathrm{SPARSE})\right)\right)$ then NP $=\mathrm{RP}$.

Proof Let $L \in R_{b}^{p}\left(R_{m}^{c o-r p}\left(R_{c}^{p}(\mathrm{SPARSE})\right)\right)$ be a solution of the promise problem (1SAT, SAT). Then, by definition, $(\forall x)[x \in 1 \mathrm{SAT} \Rightarrow(x \in L \Leftrightarrow x \in \mathrm{SAT})]$. The natural left set associated with SAT is Left (SAT) $=\left\{\langle x, w\rangle \mid x \in \mathrm{SAT}, w \in \Sigma^{l(x)}\right.$ and $\left.w \leq w_{\max }\right\}$ where $w_{\max }$ is the maximum satisfying assignment for $x$ and $l(x)$ is the number of variables in $x$. The set prefix $($ Left $(\mathrm{SAT}))=\{\langle x, y\rangle \mid(\exists z)[\langle x, y z\rangle \in \operatorname{Left}(\mathrm{SAT})]\}$ 
is easily seen to be accepted by an NP-machine that on input $\langle x, y\rangle$ guesses a truth assignment $w \geq y 0^{l(x)-|y|}$ and verfies that $w$ satisfies $x$. It is clear that $x \in 1$ SAT implies that for all $y \in \Sigma^{\leq l(x)}$, the above mentioned NP-machine has at most one accepting path on input $\langle x, y\rangle$. Let $\mathrm{g}$ be a parsimonious many-one reduction from prefix (Left(SAT)) to SAT. Then it is clear from the discussion that $x \in 1$ SAT implies $g(\langle x, y\rangle) \in 1 \mathrm{SAT}$ for all $y \in \Sigma^{\leq l(x)}$. Let $Q=\{\langle x, y\rangle \mid x \in 1$ SAT $\}$ and let $L^{\prime}=\{\langle x, y\rangle \mid g(\langle x, y\rangle) \in L\}$. Clearly $g$ many-one reduces $L^{\prime}$ to $L$.

Claim $7 L^{\prime}$ is a solution of the promise problem $(Q$, prefix (Left(SAT))).

\section{Proof of Claim}

We have to show that for every pair $\langle x, y\rangle \in Q$ it holds that $\langle x, y\rangle \in L^{\prime} \Leftrightarrow\langle x, y\rangle \in$ prefix (Left(SAT)). Since $L$ is a solution of (1SAT, SAT) and since $\langle x, y\rangle \in Q$ implies $g(\langle x, y\rangle) \in$ 1SAT, it follows that $g(\langle x, y\rangle) \in L$ if and only if $g(\langle x, y\rangle) \in$ SAT. Since $g$ many-one reduces both prefix (Left (SAT)) to SAT and $L^{\prime}$ to $L$, it follows that $\langle x, y\rangle \in$ $L^{\prime} \Leftrightarrow\langle x, y\rangle \in \operatorname{prefix}($ Left $(\mathrm{SAT}))$.

Since $L^{\prime} \leq_{m}^{p} L$ it follows that $L^{\prime} \in R_{b t t}^{p}\left(R_{m}^{c o-r p}\left(R_{c t t}^{p}(\right.\right.$ SPARSE $\left.\left.)\right)\right)$. Therefore, since $R_{m}^{c o-r p}\left(R_{c t t}^{p}(\mathrm{SPARSE})\right)$ is a set ring, $L^{\prime}$ can be written as $\bigcup_{i=1}^{k}\left(C_{2 i-1}-C_{2 i}\right)$ for sets $C_{1} \supseteq$ $C_{2} \supseteq \ldots \supseteq C_{2 k}$ in $R_{m}^{c o-r p}\left(R_{c t t}^{p}(\right.$ SPARSE $\left.)\right)$. Let $f_{i}, 1 \leq i \leq 2 k$, be co-rp reduction functions witnessing $C_{i} \in R_{m}^{c-r p}\left(R_{c t t}^{p}\right.$ (SPARSE)). Consider the algorithm described in the proof of Theorem ?? in which we use the reduction functions $f_{i}$ defined above. We claim that on input $x \in 1 \mathrm{SAT} \cap \mathrm{SAT}$ this algorithm computes with high probability the unique satisfying assignment for $x$. In order to see this, note that the algorithm on input $x \in 1 \mathrm{SAT} \cap \mathrm{SAT}$ computes query sets $f(\langle x, y, w\rangle)$ only for triples $\langle x, y, w\rangle$ for which $\langle x, y\rangle$ is in $Q$. According to Claim ?? it holds for all $\langle x, y\rangle \in Q$ that $\langle x, y\rangle \in L^{\prime} \Leftrightarrow\langle x, y\rangle \in \operatorname{prefix}($ Left(SAT)), and therefore the arguments in the proof of Theorem ?? apply. Hence there is an RP solution for the promise problem (1SAT, SAT) and by Theorem ?? it follows that NP = RP.

The following theorem concerning deterministic reductions can be similarly proved.

Theorem 5.4 If the promise problem (1SAT, SAT) has a solution in $R_{b}^{p}\left(R_{c}^{p}\right.$ (SPARSE)) then it has a solution in $\mathrm{P}$.

We need the following lemma for the next corollary.

Lemma 5.5 [?] Let $L$ be a solution of (1SAT, SAT) then Few $\subseteq \mathrm{P}^{L}$.

Proof Since Few $\subseteq \mathrm{P}^{\text {FewP }}[?]$ it suffices to show that FewP is contained in $\mathrm{P}^{L}$. Let $A$ be a set in FewP via some nondeterministic machine $M$. Let $p$ be the polynomial bounding the number of accepting paths of $M$. Consider the following NP set $B$.

$$
B=\{\langle x, i\rangle \mid M(x) \text { has at least } i \text { accepting paths }\}
$$

Let $\operatorname{acc}_{M}(x)$ denote the number of accepting paths of $M$ on input $x$. Clearly, there is an NP machine $M^{\prime}$ accepting $B$ in such a way that $M^{\prime}$ on input $\left\langle x, a c c_{M}(x)\right\rangle$ has exactly one accepting path. Then it holds that $f(\langle x, j\rangle)$ is in 1 SAT for all $j \geq \operatorname{acc}_{M}(x)$, where $f$ is a parsimonious reduction from $L\left(M^{\prime}\right)$ to SAT. Therefore $x$ is in $A$ if and only if $f(\langle x, i\rangle) \in L$ for some $i, 1 \leq i \leq p(|x|)$.

Corollary 5.6 If the promise problem (1SAT, SAT) has a solution in $R_{b}^{p}\left(R_{c}^{p}\right.$ (SPARSE)) then Few $=\mathrm{P}$. 


\section{A trade-off analysis}

It is interesting to note that the proof of Theorem ?? is constructive in the following sense: given a polynomial time truth-table condition generator $g$ witnessing prefix $(\operatorname{Left}(A))$ in $R_{b}^{p}\left(R_{c t t}^{p}(S)\right)$ for a sparse set $S$, the conjunctive query sets $f_{i}(\langle x, y\rangle)$ can be computed from the truth-table condition $g(\langle x, y\rangle)$ by a polynomial time algorithm (as can be derived from a general result in [?] that applies to various set rings). Therefore, given an FP transducer computing $g$ and a polynomial bound on the census of $S$, we get a polynomial time decision procedure for $A$. The question arises how the running time of that algorithm is influenced if the number $k$ of the conjunctive queries produced by $g$ is a function $k(n)$ depending on the length $n$ of $x$ rather than a constant, and if the census of $S$ is allowed to be an arbitrary function.

In the next theorem we precisely analyze the running time of the algorithm in terms of the functions $k$ and census $_{S}$, assuming that $g$ directly generates truth-table conditions suitable for our algorithm.

More precisely, we assume that $g$ is a truth-table reduction of the following type which we call Hausdorff reduction and which is a variation of the reducibility defined by K.W. Wagner in [?].

Definition 6.1 Let $h\left(x_{1}, x_{2}, \ldots, x_{k}\right)$ be the boolean formula $\oplus_{i=1}^{k}\left(\wedge_{j=1}^{i} x_{j}\right)$, where $\oplus$ denotes the parity operator. We say that a set $A$ is $k(n)$-Hausdorff reducible to $B$ if $A$ is truth-table reducible to $B$ via the boolean function $h\left(x_{1}, x_{2}, \ldots, x_{k(n)}\right)$, i.e. there is a polynomial time computable query generator $g$ such that for all $x, x \in A \Leftrightarrow$ $h\left(\chi_{B}\left(y_{1}\right), \cdots, \chi_{B}\left(y_{k(|x|)}\right)\right)=1$ where $g(x)=\left\langle y_{1}, \cdots, y_{k(|x|)}\right\rangle$.

Observe that $A \in R_{b}^{p}\left(R_{c t t}^{p}\right.$ (SPARSE)) if and only if $A$ is bounded Hausdorff reducible to some set in $R_{c t t}^{p}$ (SPARSE). We now state the trade-off result.

Theorem 6.2 If $B$ is a set of density bounded by an FP function $c_{B}$ and if some NP complete set is polynomial time reducible to a set in $R_{c t t}^{p}(B)$ by a $k(n)$-Hausdorff reduction, then NP $\subseteq \bigcup_{j \geq 0} \operatorname{DTIME}\left(n^{j} \cdot c_{B}\left(n^{j}\right)^{O\left(k\left(n^{j}\right)\right)}\right)$.

Proof Suppose that a set $B$ as in the statement exists. Then for $A \in \mathrm{NP}$ the set $\operatorname{prefix}(\operatorname{Left}(A))$ is reducible via a $k\left(n^{O(1)}\right)$-Hausdorff reduction to a set in $R_{c t t}^{p}(B)$. It is not hard to see that there is an FP function $f$ such that for all $x, y$

$$
\langle x, y\rangle \in \operatorname{prefix}(\operatorname{Left}(A)) \Leftrightarrow \max \{i \mid 1 \leq i \leq k(|x|), f(\langle i, x, y\rangle) \subseteq B\} \text { is odd }
$$

Observe that the function $f$ can be used instead of the conjunctive reduction functions $f_{i}$ in the proof of Theorem ??. Therefore with a minor modification we can use the algorithm described in the proof of Theorem ?? to compute $w_{\max }$ on input $x \in A$. It only remains to accurately analyze the running time of the algorithm taking into account the growth rate of the function $k$ and of the density of $B$.

First note that the depth of recursive calls made by procedure PRUNE is $k\left(n^{O(1)}\right)$. Next we examine the calls to SEARCH-LEFT and SEARCH-RIGHT. The size of the set returned by each such call is bounded by $c_{B}\left(n^{O(1)}\right)$. Since the pruned subset of $N$ returned by PRUNE is constructed by taking prefixes corresponding to all subsets returned by SEARCH-LEFT in all the recursive calls by PRUNE, the size of this pruned subset is bounded by $c_{B}\left(n^{O(1)}\right)^{\left.O\left(k_{(n} O(1)\right)\right)}$. The running time of each call is bounded by 
$|N| \cdot n^{O(1)}$. Furthermore, the total number of calls made by PRUNE to both SEARCHRIGHT and SEARCH-LEFT (including the recursive calls) is bounded by $|N| \cdot 2 k\left(n^{O(1)}\right)$. This implies that the running time of one call to PRUNE is bounded by $k\left(n^{O(1)}\right) \cdot n^{O(1)}$. $c_{B}\left(n^{O(1)}\right)^{O\left(k\left(n^{O(1)}\right)\right)}$. The overall running time of the algorithm has only an additional polynomial factor. This completes the proof.

An interesting point in the above result is that the actual number of queries in the conjunctive reduction plays no real role in the trade-off. The next corollary to the above theorem is similar to a result in [?] concerning $f(n)$-tt hard sets of certain densities for NP.

Corollary 6.3 If $B$ is a set of density $O(\log n)$ such that an NP complete set is reducible to a set in $R_{c t t}^{p}(B)$ by a $O(\log n / \log \log n)$-Hausdorff reduction then $\mathrm{P}=\mathrm{NP}$.

Corollary 6.4 If an NP complete set is reducible to a set in $R_{c t t}^{p}(\mathrm{SPARSE})$ by a $O(\log n)$ Hausdorff reduction then $\mathrm{NP} \subseteq \operatorname{DTIME}\left(2^{O\left(\log ^{2} n\right)}\right)$.

We now give a trade-off analysis for the algorithm in Theorem ??

Theorem 6.5 If $B$ is a set of density bounded by an $\mathrm{FP}$ function $c_{B}$ and if some NP complete set is polynomial-time reducible to a set in $R_{m}^{c o-r p}\left(R_{c t t}^{p}(B)\right)$ by a $k(n)$-Hausdorff reduction then $\mathrm{NP} \subseteq \bigcup_{j \geq 0} \operatorname{RTIME}\left(n^{j} \cdot c_{B}\left(n^{j}\right)^{O\left(k\left(n^{j}\right)\right)}\right)$.

Proof Follows easily (as done in Theorem ??) from a simple analysis of the corresponding algorithm in the proof of Theorem ??. One thing to be noted is that, for amplification, the polynomial $p$ in the proof of Theorem ?? can be again chosen so that $\left(1-2^{-p(|x|)}\right)^{r(|x|) \cdot 2 k(n)} \geq 3 / 4$, since $k(n)$ is anyway bounded by some polynomial.

Corollary 6.6 If $B$ is a set of density $O(\log n)$ such that an NP complete set is reducible to a set in $R_{m}^{\text {co-rp }}\left(R_{c t t}^{p}(B)\right)$ by an $O(\log n / \log \log n)$-Hausdorff reduction then $\mathrm{NP}=\mathrm{RP}$.

Corollary 6.7 If an NP complete set is reducible to a set in $R_{m}^{c o-r p}\left(R_{c t t}^{p}(\mathrm{SPARSE})\right)$ by an $O(\log n)$-Hausdorff reduction then $\mathrm{NP} \subseteq \operatorname{RTIME}\left(2^{O\left(\log ^{2} n\right)}\right)$.

\section{Nondeterministic reductions to sparse sets}

In this section we consider classes of sets reducible to sparse and tally sets via polynomial time nondeterministic reductions. We show that nondeterministic polynomial time manyone reductions to sparse sets are as powerful as nondeterministic Turing reductions to sparse sets. On the other hand, nondeterministic polynomial time many-one reductions to co-sparse sets are much weaker. We substantiate this claim by proving, applying essentially Kadin's census technique [?], that if coNP is nondeterministically polynomial time many-one reducible to a co-sparse set then $\mathrm{PH}=\Theta_{2}^{p}$. A similar result for sparse sets is unlikely since it would imply that the Karp/Lipton/Sipser result [?] that $\mathrm{NP} \subseteq \mathrm{P} /$ poly implies $\mathrm{PH}=\Sigma_{2}^{p}$ could be improved (known to be impossible in relativized worlds [?]).

The following definitions of nondeterministic polynomial time reductions are due to Ladner, Lynch and Selman [?]. 
1. A set $A$ is polynomial time nondeterministically many-one reducible to a set $B$ (denoted $A \leq_{m}^{n p} B$ ) if there exists a polynomial time nondeterministic Turing machine $M$ such that for every $x \in \Sigma^{*}, M(x)$ outputs a string along each computation path, and, $x \in A$ iff $M(x)$ outputs some string in $B$.

D. A set $A$ is polynomial time nondeterministically Turing reducible to a set $B$ (denoted $\left.A \leq_{T}^{n p} B\right)$ if there exists a polynomial time nondeterministic oracle Turing machine $M$ accepting $A$ with oracle $B$, i.e. $A=L(M, B)$.

Our first result in this section is the equality $R_{m}^{n p}$ (SPARSE) $=R_{T}^{n p}$ (SPARSE). Indeed, we show that every set in $R_{T}^{n p}$ (SPARSE) nondeterministically many-one reduces to very sparse sets (in the sense that they contain at most one string of each length). Note that in [?] it is shown, using the inclusion $R_{b}^{p}$ (SPARSE) $\subseteq R_{d t t}^{p}\left(\right.$ SPARSE), that $R_{c}^{n p}$ (SPARSE) $=$ $R_{T}^{n p}$ (SPARSE) and that $R_{m}^{n p}(\mathrm{SPARSE})=R_{d t t}^{n p}(\mathrm{SPARSE})$.

Theorem 7.2 $R_{m}^{n p}(\mathrm{SPARSE})=R_{T}^{n p}(\mathrm{SPARSE})$

Proof Let $A=L(M, S) \in R_{T}^{n p}(S)$ for a sparse set $S$ and a nondeterministic Turing machine $M$. Let $p$ be a polynomial bounding the running time of $M$, and let $q$ be a polynomial bounding the census of $S$. Clearly, each query generated by $M$ along any computation path on an input $x$ is in $\Sigma \leq p(|x|)$. We define a sparse set $S^{\prime}$ to which $A$ can be nondeterminstically many-one reduced in polynomial time.

$$
S^{\prime}=\left\{\left\langle 0^{n}, y_{1}, y_{2}, \cdots, y_{r}\right\rangle \mid y_{1}<\cdots<y_{r} \text { and }\left\{y_{1}, \cdots, y_{r}\right\}=S^{\leq n}\right\}
$$

Note that $S^{\prime}$ has for each length at most one element. Consider the following NP-machine $M^{\prime}$.

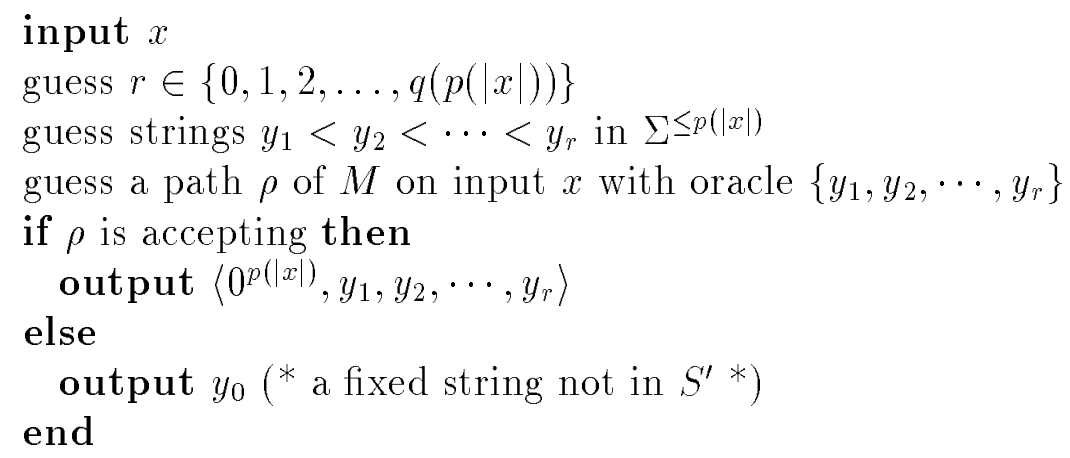

It is not hard to see that $M^{\prime}$ witnesses $A \in R_{m}^{n p}\left(S^{\prime}\right)$. This completes the proof.

Related to the broad question discussed in Sections ?? and ?? whether NP can have hard sparse sets with respect to deterministic or randomized polynomial time reductions (of different kinds) one can ask similar questions with respect to nondeterministic polynomial time reductions.

1. Can coNP have sparse hard sets under nondeterministic polynomial time many-one reductions? 
2. Can coNP have co-sparse hard sets under nondeterministic polynomial time manyone reductions?

Such questions have been implicitly considered in the literature with regard to nonuniform classes. Balcázar and Schöning [?] show that coNP $\subseteq \mathrm{NP} / \log$ implies $\mathrm{PH}=\Theta_{2}^{p}$. Similarly it is known that coNP $\subseteq \mathrm{NP} /$ poly implies $\mathrm{PH}=\Sigma_{3}^{p}$ [?].

Theorem 7.3 If coNP $\subseteq R_{m}^{n p}\left(\right.$ co-SPARSE) then $\mathrm{PH}=\Theta_{2}^{p}$.

Proof Let $A$ be a complete set for coNP that is many-one reducible via a nondeterministic polynomial time Turing machine $M$ to the complement $\bar{S}$ of a sparse set $S$. We define a sparse set $S^{\prime} \in \mathrm{NP}$ such that $A \in \mathrm{NP}^{S^{\prime}}$. This proves the theorem since by Kadin's result [?] it follows that $\mathrm{PH}=\Theta_{2}^{p}$. $S^{\prime}$ contains all strings that are provably not in $\bar{S}$.

$$
S^{\prime}=\{y \mid \exists x \in \bar{A}: M \text { on input } x \text { outputs } y\}
$$

It is clear that $S^{\prime} \subseteq S$ and it remains to show that $A \in \mathrm{NP}^{S^{\prime}}$. Consider the following nondeterminstic oracle machine $M^{\prime}$.

On input $x, M^{\prime}$ simulates $M$ on $x$. If $M$ outputs $y$ at the end of the simulated computation path then $M^{\prime}$ queries oracle $S^{\prime}$ for $y$ and accepts if and only if $y \notin S^{\prime}$.

If $x \in A$ then some output $y$ of $M$ on input $x$ is in $\bar{S}$. Since $S^{\prime} \subseteq S$, it holds that $y \notin S^{\prime}$ and hence $x \in L\left(M^{\prime}, S^{\prime}\right)$. If $x \notin A$ then every output of $M$ on input $x$ is in $S^{\prime}$ and therefore $x \notin L\left(M^{\prime}, S^{\prime}\right)$. Therefore $L\left(M^{\prime}, S^{\prime}\right)=A$.

From Theorem ?? we know that $R_{m}^{n p}$ (SPARSE) $=\mathrm{NP} /$ poly, hence it appears difficult to get a comparable collapse of $\mathrm{PH}$ as in Theorem ?? under the assumption that coNP $\subseteq$ $R_{m}^{n p}$ (SPARSE).

Finally, we have a result similar to Theorem ?? for nondeterministic reductions.

Theorem 7.4 If $\Sigma_{2}^{p} \subseteq R_{b}^{p}\left(R_{m}^{n p}(\right.$ co-SPARSE $\left.)\right)$ then $\mathrm{PH}=\Delta_{2}^{p}$.

\section{References}

[AM77] L. Adleman and K. Manders. Reducibility, randomness and intractability, In Proceedings of the 9th ACM Sympos. Theory of Computing 151-163.

[AHH+92] V. Arvind, Y. Han, L. Hemachandra, J. Köbler, A. Lozano, M. Mundhenk, M. Ogiwara, U. Schöning, R. Silvestri, and T. Thierauf. Reductions to sets of low information content. In Proceedings of the 19th International Colloquium on Automata, Languages, and Programming. To appear.

[AHOW] E. Allender, L. Hemachandra, M. Ogiwara, and O. Watanabe. Relating equivalence and reducibility to sparse sets. SIAM Journal on Computing. To appear. Preliminary version appears as [?].

[AHOW91] E. Allender, L. Hemachandra, M. Ogiwara, and O. Watanabe. Relating equivalence and reducibility to sparse sets. Proceeding 6th Structure in Complexity Theory Conference, 1991. 
[ABG90] A. Amir, R. Beigel, and W. Gasarch. Some connections between bounded query classes and non-uniform complexity. In Proceedings of the 5th Structure in Complexity Theory Conference, pages 232-243. IEEE Computer Society Press, July 1990.

[Ba190] J.L. Balcázar. Self-reducibility. Journal of Computer and System Sciences, 41(3):367-388, 1990.

[BBS86] J. Balcázar, R. Book, and U. Schöning. Sparse sets, lowness and highness. SIAM J. Comput., 15:739-745, 1986.

[BaSchö88] J.L. Balcázar and U. Schöning. Logarithmic advice classes. Bericht 9/88, Informatik, EWH Koblenz, to appear in Theoretical Computer Science.

[BeGiHe90] R. Beigel, J. Gill, U. Hertrampf. Counting classes: Thresholds, parity, mods, and fewness. In Proceedings 7th Symposium on Theoretical Aspects of Computer Science, Lecture Notes in Computer Science 415 (1990), 49-57.

[Ber78] P. Berman. Relationship between density and deterministic complexity of NPcomplete languages. In Proceedings of the 5th International Colloquium on Automata, Languages, and Programming, pages 63-71. Springer-Verlag Lecture Notes in Computer Science \#62, 1978.

[BH77] L. Berman and J. Hartmanis. On isomorphisms and density of NP and other complete sets. SIAM Journal on Computing, 6(2):305-322, 1977.

[BLS92] H. Buhrman, L. Longpré, and E. Spaan. personal communication, 1992.

[CH91] J. Cai and L. Hemachandra. A note on enumerative counting. Information Processing Letters, 38(4):215-219, 1991.

[ESY84] S. Even, A. Selman, and Y. Yacobi. The complexity of promise problems with applications to public-key cryptography. Information and Control, 61:114-133, 1984.

[For79] S. Fortune. A note on sparse complete sets. SIAM Journal on Computing, 8(3):431-433, 1979.

[Hau14] F. Hausdorff. Grundzüge der Mengenlehre. Leipzig, 1914.

[HL91] S. Homer and L. Longpré. On reductions of NP sets to sparse sets. In Proceedings of the 6th Structure in Complexity Theory Conference, pages 79-88. IEEE Computer Society Press, June/July 1991.

[IM89] N. Immerman and S. Mahaney. Relativizing relativized computations. Theoretical Computer Science, 68:267-276, 1989.

[Kad88] J. Kadin. Restricted Turing reducibilities and the structure of the polynomial time hierarchy. PhD thesis, Cornell University, 1988.

[KL80] R. Karp and R. Lipton. Some connections between nonuniform and uniform complexity classes. In Proceedings of the 12th ACM Symposium on Theory of Computing, pages 302-309, April 1980. 
[KSTT89] J. Köbler, U. Schöning, S. Toda, J. Torán. Turing machines with few accepting computations and low sets for PP. In Proceedings of the 4 th Structure in Complexity Theory Conference, pages 208-215. IEEE Computer Society Press, June/July 1989.

[KSTT] J. Köbler, U. Schöning, S. Toda, J. Torán. Turing machines with few accepting computations and low sets for PP. Journal of Computer and System Sciences. To appear. Preliminary version appears as [?].

[LLS75] R. Ladner, N. Lynch, and A. Selman. A comparison of polynomial time reducibilities. Theoretical Computer Science, 1(2):103-124, 1975.

[Mah82] S. Mahaney. Sparse complete sets for NP: Solution of a conjecture of Berman and Hartmanis. Journal of Computer and System Sciences, 25(2):130-143, 1982.

[OL91] M. Ogiwara and A. Lozano. On one query self-reducible sets. In Proceedings of the 6th Structure in Complexity Theory Conference, pages 139-151. IEEE Computer Society Press, June/July 1991.

[OW91] M. Ogiwara and O. Watanabe. On polynomial-time bounded truth-table reducibility of NP sets to sparse sets. SIAM Journal on Computing, 20(3):471483, June 1991.

[RR92] D. Ranjan and P. Rohatgi. Randomized reductions to sparse sets. In Proceedings 1th Structure in Complexity Theory Conference. To appear.

[VV86] L.G. Valiant and V.V Vazirani. NP is as easy as detecting unique solutions. Theoretical Computer Science, 47(1):85-93, 1986.

[Wag87] K.W. Wagner. More complicated questions about maxima and minima, and some closures of NP. Theoretical Computer Science, 51(1):53-80, 1987.

[WW85] G. Wechsung and K.W. Wagner. On the boolean closure of NP. Manuscript. (Extended abstract by: G. Wechsung, On the boolean closure of NP, in Proc. 1985 International Conference on Fundamentals of Computation Theory, pages 485-493. Lecture Notes in Computer Science, Springer-Verlag, 1985.) 